\title{
Pinning the Order: The Nature of Quantum Criticality in the Hubbard Model on Honeycomb Lattice
}

\author{
Fakher F. Assaad ${ }^{1,3}$ and Igor F. Herbut ${ }^{2,3}$ \\ ${ }^{1}$ Institut für Theoretische Physik und Astrophysik, Universität Würzburg, Am Hubland, D-97074 Würzburg, Germany \\ ${ }^{2}$ Department of Physics, Simon Fraser University, Burnaby, British Columbia V5A 1S6, Canada \\ ${ }^{3}$ Max-Planck-Institut für Physik Komplexer Systeme, Nöthnitzer Strasse 38, 01187 Dresden, Germany
}

(Received 1 May 2013; published 26 August 2013)

\begin{abstract}
In numerical simulations, spontaneously broken symmetry is often detected by computing two-point correlation functions of the appropriate local order parameter. This approach, however, computes the square of the local order parameter, and so when it is small, very large system sizes at high precisions are required to obtain reliable results. Alternatively, one can pin the order by introducing a local symmetrybreaking field and then measure the induced local order parameter infinitely far from the pinning center. The method is tested here at length for the Hubbard model on honeycomb lattice, within the realm of the projective auxiliary-field quantum Monte Carlo algorithm. With our enhanced resolution, we find a direct and continuous quantum phase transition between the semimetallic and the insulating antiferromagnetic states with increase of the interaction. The single-particle gap, measured in units of Hubbard $U$, tracks the staggered magnetization. An excellent data collapse is obtained by finite-size scaling, with the values of the critical exponents in accord with the Gross-Neveu universality class of the transition.
\end{abstract}

DOI: 10.1103/PhysRevX.3.031010

\section{INTRODUCTION}

Detecting spontaneous symmetry-broken phases in numerical simulations often relies on the measure of correlation function. For instance, the magnetically ordered phase is characterized by long-ranged spin-spin correlations, whereas the superconducting state exhibits long-ranged pair correlations in the appropriate symmetry channel. A fundamental caveat with such an approach is that one measures the square of the order parameter. If the latter quantity is small, the quantity one attempts to obtain by extrapolating numerical data to the thermodynamic limit is quadratically smaller. As a consequence, very large system sizes at high precision are required in addition to an appropriate finite-size extrapolation formula.

The aim of this article is twofold. We will document a simple and very efficient alternative method to detect magnetically ordered phases in SU(2)-invariant Hubbard-type models in the realm of projective quantum Monte Carlo methods. With the enhanced resolution, we will revisit the semimetal-to-insulator transition on graphene's honeycomb lattice, which has recently been under considerable debate $[1,2]$.

Honeycomb lattice is a bipartite, nonfrustrated lattice, which at half-filling and small Hubbard repulsion $U$ hosts the semimetallic state of electrons, as in graphene. When the repulsion is increased, one eventually expects a phase

Published by the American Physical Society under the terms of the Creative Commons Attribution 3.0 License. Further distribution of this work must maintain attribution to the author(s) and the published article's title, journal citation, and DOI.
Subject Areas: Computational Physics, Mesoscopics, Strongly Correlated Materials

transition into an insulating state with antiferromagnetic order [3-5] which, because of gapless Dirac fermionic excitations being present on the semimetallic side, should belong to a particular Gross-Neveu universality class [5,6].

Starting from the strong coupling limit and noting that the insulator-to-metal transition occurs at values of the Hubbard interaction lesser than the bandwidth allows for the proliferation of higher-order ring-exchange terms in an effective spin model aimed at describing the magnetic insulating state in the vicinity of the transition [7]. This point of view opens the possibility that the melting of the magnetic order is unrelated to the metal-insulator transition. Recent quantum Monte Carlo calculations [1] suggested that there is an intermediate spin-liquid phase with a single-particle gap but no magnetic ordering, separating the semimetal and magnetic insulator. Similar results have been put forward for the related $\pi$-flux model on the square lattice [8]. The results of Ref. [1] have been challenged by recent studies. Entropy calculations do not favor groundstate degeneracy, as expected for the $Z_{2}$ spin liquid [9]. Moreover, Ref. [2] shows that extrapolating from significantly larger system sizes would suggest almost complete disappearance of the spin liquid from the phase diagram. The latter conclusion is reinforced here, where we find excellent data collapse and identical finite-size scaling of both the single-particle gap and staggered magnetization, with the distinct values of critical exponents, in accord with the Gross-Neveu universality class $[5,6]$.

From the technical point of view, our approach is very similar in spirit to an approach considered in Ref. [10]. By introducing a local magnetic field at, say, the origin, we explicitly break the SU(2) spin symmetry. In the presence 
of long-range order and in the thermodynamic limit, any field will pin the order along the direction of the external field. Thereby, order can be detected by computing directly the magnetization infinitely far from the pinning field. The upside of such an approach is that one measures directly the order parameter rather than its square. This procedure amounts to evaluating a single-particle quantity, which is often much more stable than correlation functions. The downsides are threefold. One explicitly breaks SU(2) spin symmetry such that spin sectors mix and it becomes computationally more expensive to reach the ground state. Since the computational cost scales linearly with the projection parameter, this problem is tractable. The second difficulty lies in the ordering of limits. To obtain results that are independent on the magnitude of the pinning field, it is important to first take the thermodynamic limit and then the limit of infinite distance from the pinning field. In a practical implementation, this ordering of limits has, as a consequence, some leftover dependence of the magnetization on the magnitude of the pinning field. This feature is particularly visible when the pinning field is small. The final drawback is that it is not always possible to introduce a pinning field without generating a negative sign problem. For instance, in the Kane-Mele-Hubbard model [11-13], the spin order lies in the $x-y$ plane. Adding a magnetic field along this quantization axis introduces a sign problem. On the other hand, the method is applicable to $\mathrm{SU}(N)$ symmetric Hubbard-Heisenberg models [14].

The organization and main results of the article are the following. We focus on the Hubbard model on honeycomb lattice at the filling one-half, for which the presence of an intermediate spin-liquid phase has been controversial [1,2]. After introducing and testing the approach in the next section, we provide a phase diagram of the Hubbard model in Sec. III. The data point to the fact that the staggered moment follows rather precisely the single-particle gap, when the gap is measured in the natural units of the Hubbard $U$, suggesting a direct quantum phase transition between the semimetallic and the insulating antiferromagnetic phases. Furthermore, an excellent finite-size scaling of the data for both the staggered magnetization and the single-particle gap is found by assuming the values of the critical exponents $\beta=0.79$ and $\nu=0.88$. These values are the ones found in the first-order expansion for the Gross-Neveu-Yukawa field theory of this quantum phase transition [6], around its upper critical (spatial) dimension of three. Altogether, the data strongly support the existence of a single quantum critical point separating the semimetallic and the insulating antiferromagnetic phases of the Hubbard model, with the quantum criticality belonging to the Gross-Neveu universality class [5].

\section{MODEL AND METHOD}

As in Ref. [1], we will consider the half-filled Hubbard model on the honeycomb lattice
$H_{t U}=-t \sum_{\langle\mathbf{i}, \mathbf{j}\rangle, \sigma} c_{\mathbf{i}, \sigma}^{\dagger} c_{\mathbf{j}, \sigma}+U \sum_{\mathbf{i}}\left(n_{\mathbf{i}, \uparrow}-1 / 2\right)\left(n_{\mathbf{i}, \downarrow}-1 / 2\right)$.

The hopping is restricted to nearest neighbors, so that the bipartite nature of the lattice allows us to avoid the negative sign problem.

Generically, to detect antiferromagnetic ordering, we compute spin-spin correlations:

$$
m=\lim _{L \rightarrow \infty} \sqrt{\frac{1}{N} \sum_{\mathbf{i}=1}^{N} e^{i \mathbf{Q} \cdot \mathbf{i}}\left\langle\mathbf{S}_{\mathbf{0}} \cdot \mathbf{S}_{\mathbf{i}}\right\rangle_{H_{t U}}}
$$

Here, $N=2 L^{2}$ corresponds to the number of orbitals, and $L$ is the linear length of the lattice. A finite value of $m$ signalizes long-range order and is equivalent to spontaneous symmetry breaking. In particular, including a magnetic-field term with an appropriate Fourier component

$$
H_{h}=h \sum_{\mathbf{i}} e^{i \mathbf{Q} \cdot \mathbf{i}} S_{i}^{z}
$$

gives

$$
m=\lim _{h \rightarrow 0} \lim _{L \rightarrow \infty} \frac{1}{L^{2}} \sum_{\mathbf{i}} e^{i \mathbf{Q} \cdot \mathbf{i}}\left\langle S_{\mathbf{i}}^{z}\right\rangle_{H_{t U}+H_{h}} .
$$

The ordering of limits is crucial. One first has to take the thermodynamic limit to allow for the collapse of Anderson's tower of states and then the limit of vanishing magnetic field $h$. Such an approach was, for instance, used in Ref. [15].

It is more convenient to consider a local field since, as we will see below, this trick lifts the burden of taking the limit $h \rightarrow 0$ numerically. The local pinning field is given by the term

$$
H_{\mathrm{loc}}=h_{0} S_{\mathbf{0}}^{z}
$$

in the Hamiltonian. Using the representation $\delta_{\mathbf{i}, \mathbf{0}}=$ $\frac{1}{L^{2}} \sum_{\mathbf{q}} e^{i \mathbf{q} \cdot \mathbf{i}}$ of the Kronecker symbol shows that each Fourier component comes with an amplitude $h_{0} / L^{2}$, so that taking the thermodynamic limit is equivalent to taking the amplitude of the relevant Fourier component to zero. With the local field construction, the appropriate ordering of limits for an $L \times L$ lattice reads

$$
m=\lim _{\mathbf{i} \rightarrow \infty} \lim _{L \rightarrow \infty} e^{i \mathbf{Q} \cdot \mathbf{i}}\left\langle S_{\mathbf{i}}^{z}\right\rangle_{H_{t U}}+H_{1 \mathrm{loc}} \cdot
$$

That is, one first has to take the thermodynamic limitagain to guarantee the collapse of the tower of states in the presence of long-range order-and only then can one take the distance from the pinning center to infinity [16]. In other words, the distance from the pinning center sets an energy scale that has to be larger than the finite-size spin gap. As an efficient estimator for the evaluation of the ordered moment, we thus propose 


$$
m=\lim _{L \rightarrow \infty} \frac{1}{L^{2}} \sum_{\mathbf{i}} e^{i \mathbf{Q} \cdot \mathbf{i}}\left\langle S_{\mathbf{i}}^{z}\right\rangle_{H_{t U}}+H_{\mathrm{loc}} \cdot
$$

We have tested the above approach for the Hubbard model on the honeycomb lattice. Ground-state calculations were carried out with the projective auxiliary-field quantum Monte Carlo (QMC) algorithm, which is based on the equation

$$
\langle O\rangle_{H}=\lim _{\theta \rightarrow \infty} \frac{\left\langle\Psi_{T}\left|e^{-\theta H / 2} O e^{-\theta H / 2}\right| \Psi_{T}\right\rangle}{\left\langle\Psi_{T}\left|e^{-\theta H}\right| \Psi_{T}\right\rangle} .
$$

Here, $\theta$ is a projection parameter, and the trial wave function is required to be nonorthogonal to the ground state. For $H=H_{t U}+H_{\mathrm{loc}}$, the inclusion of the magnetic field does not generate a negative sign problem. We have chosen the trial wave function to be the ground state of the noninteracting Hamiltonian $H_{T}=H_{t}+H_{\mathrm{loc}}$ in the $S^{z}=0$ sector. The implementation of the algorithm follows closely Refs. [1,17]. The major difference is the use of a symmetric Trotter breakup, which ensures the hermiticity of the imaginary time propagator for any value of the time discretization $\Delta \tau$. It also leads to smaller systematic errors.

Figure 1(a) plots the local moment at $U / t=5$ using different methods. In this case, magnetic ordering is robust, such that various approaches can be compared. The data set at $h_{0}=0$ corresponds to the correlation functions of Eq. (2). For this set of runs, we use a spin-singlet trial wave function, and the projection parameter $\theta t=40$ suffices to guarantee convergence to the ground state. This quick convergence stems from the fact that the trial wave function is orthogonal to the low-lying spin excitations [18]. The runs at finite values of the pinning field correspond to the quantity of Eq. (7). In the presence of a finite pinning field, $\mathrm{SU}(2)$ spin symmetry is broken and the trial wave function overlaps with all spin sectors. Consequently, a large value of the projection parameter $\theta t=320$ is required to guarantee convergence to the ground state within the quoted accuracy. Note that the CPU time scales only linearly with the projection parameter, so that such large projection parameters are still numerically tractable. It is also worth pointing out that the observable of Eq. (7) corresponds to a single-particle quantity and shows very little fluctuation. As is evident in Fig. 1(a), finite-size effects are strongly dependent on the specific choice of the pinning field. Nevertheless, convergence to values consistent with the generic approach based on Eq. (2) is obtained for relatively large values of the pinning field. If the pinning field is chosen too small, larger lattices are apparently required to ensure that the finite-size spin gap, set by $v / L$ with $v$ the spin-wave velocity, is smaller than the energy scale set by the pinning field. This expectation is confirmed by the data, which show a systematic upturn as a function of the system size for smaller values of the pinning field. Such a nonmonotonic finite-size behavior complicates a finite-size scaling analysis. For this reason,
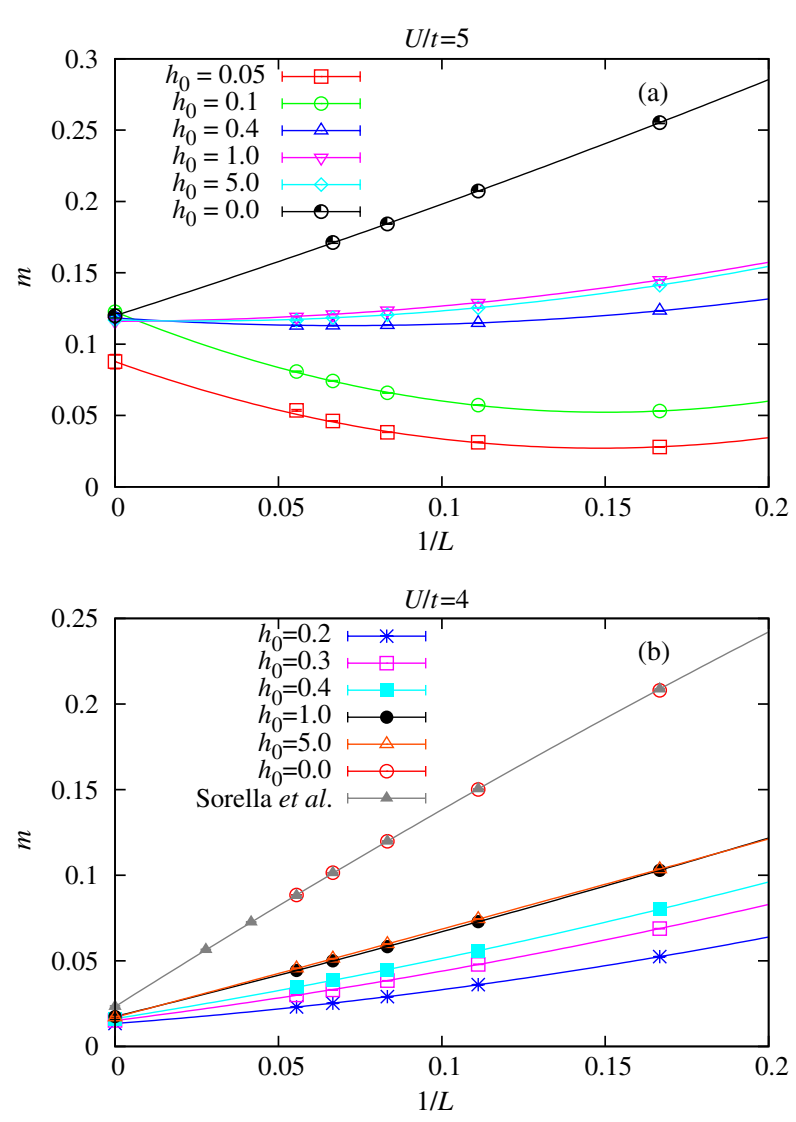

FIG. 1. Comparison of the pinning-field and correlationfunction approaches to determine the staggered moment at (a) $U / t=5$ and (b) $U / t=4$. The data sets at $h_{0}=0$ correspond to the correlations functions, and values of $\theta t=40$ are sufficient to converge to the ground state. For nonvanishing pinning fields, projection parameters $\theta t=320$ are required to guarantee convergence. We use $\Delta \tau t=0.1$, which for the symmetric Trotter decomposition yields converged results within our numerical accuracy. At $U / t=4$, comparison with the results of Ref. [2] shows excellent agreement. Lines corresponds to least-squares fits to the form $a+b / L+c / L^{2}$.

we propose to use a relatively large value of the pinning field [19].

At $U / t=4$, the local moment is smaller and hard to detect. The $h_{0}=0$ data set of Fig. 1(b) compares our results for the correlation function of Eq. (2) to those of Ref. [2]. As is apparent, the agreement up to our largest lattice size $L=18$ is remarkable. Without the largest lattice sizes $L=24$ and $L=36$, extrapolation to the thermodynamic limit is hard because of the downward turn present in the finite-size results. The data sets stemming from the pinning-field approach provide an alternative perspective and, on the whole, confirm the result of Ref. [2]. For the considered field range, there is considerable scatter in the finite-size results, but nevertheless the extrapolation to the thermodynamic limit seems to be field independent, as expected from the above considerations.

We conclude this section by mentioning that we have tested the approach for the noninteracting case and the 
method successfully demonstrates the absence of longrange magnetic order. Hence, both for critical states as well as for magnetically ordered phases, the pinning-field approach does provide an efficient tool. Further testing of this approach for Heisenberg bilayers is presently in progress [20].

\section{PHASE DIAGRAM OF THE HUBBARD MODEL ON HONEYCOMB LATTICE}

We have used the above approach to revisit the magnetic phase diagram of the Hubbard model on the honeycomb lattice. At weak couplings, the model is known to have a stable semimetallic state. In the strong coupling limit and because of the absence of frustration, an antiferromagnetic Mott insulator is present. The nature of the transition between these two states has been studied in the past [3-5] and is presently controversial [1,2].
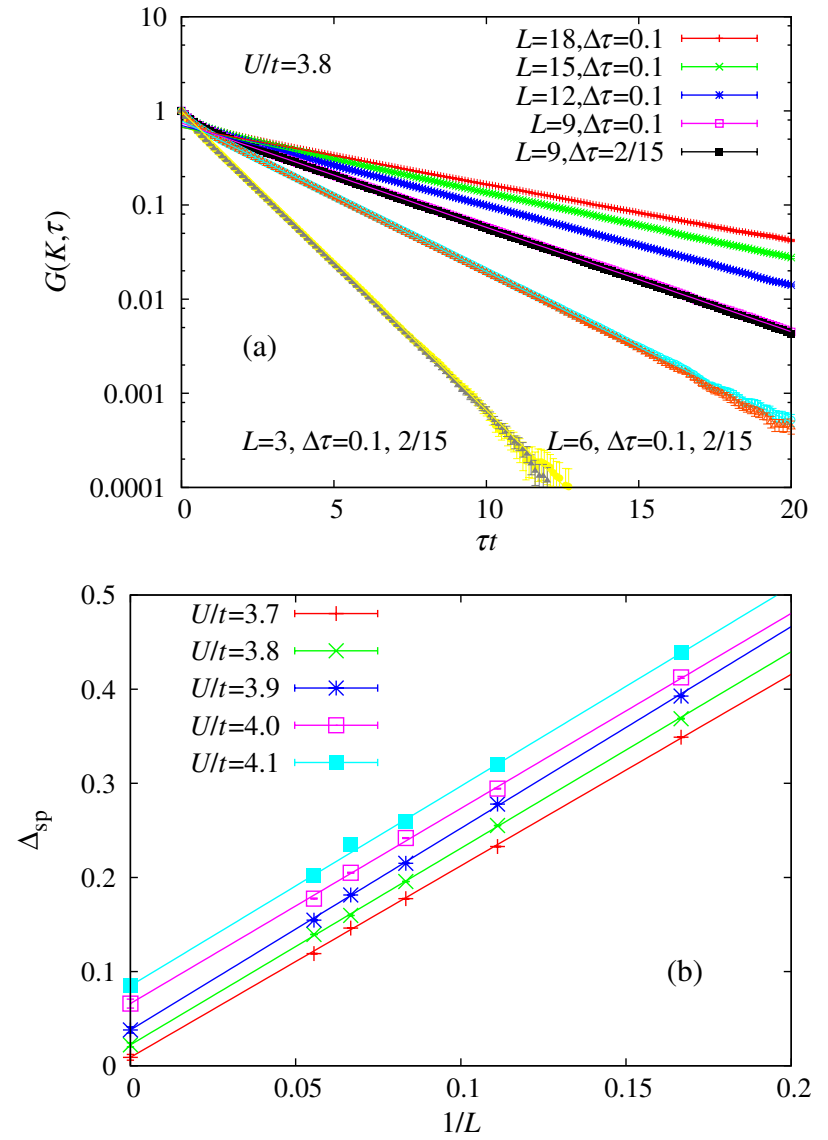

FIG. 2. Single-particle gap. (a) The raw data at $U / t=3.8$. As is apparent, the systematic error stemming from the finite Trotter step is negligible within our accuracy. The data support a large imaginary time range consistent with a single exponential decay. Lines are least-squares fits of the tail of the imaginary time Green function to the form $Z e^{-\Delta_{\text {sp }} \tau}$. Here, $Z$ corresponds to the single-particle residue and $\Delta_{\mathrm{sp}}$ to the single-particle gap. (b) Size dependence and extrapolation of the single-particle gap.

\section{A. Single-particle gap}

To pin down the coupling strength beyond which the single-particle gap opens, we have repeated calculations for the time-displaced single-particle imaginary time Green function at the nodal point: $G(K, \tau)=$ $\sum_{\sigma}\left\langle c_{\mathbf{K}, \sigma}^{\dagger}(\tau) c_{\mathbf{K}, \sigma}(\tau=0)\right\rangle$. As is evident in Fig. 2 and with the symmetric Trotter decomposition, the Trotter systematic error is negligible within our accuracy. Fitting the data to an exponential form allows us to extract the singleparticle gap, which we plot as a function of system size in Fig. 2. Assuming a polynomial form for the extrapolation to the thermodynamic limit, we find a small but finite single-particle gap for $U / t \geq 3.7$. This finding is particularly interesting when compared to the results of Sorella et al. [2] that at $U / t=3.8$ point to the absence of longrange magnetic order. This result can be taken as an indication for a possible intermediate phase. However, the analysis that we present below suggests a different interpretation.

\section{B. Magnetization from pinning fields}

We have used the pinning-field approach to compute the staggered moment as a function of $U / t$. The results at $h_{0}=5 t$ are reported in Fig. 3. The extrapolation to the thermodynamic limit is carried out using a polynomial scaling up to second order in $1 / L$. Figure 4 plots the soobtained staggered moment for two choices of the pinning field as well as the single-particle gap. Several comments are in order.

(i) Within our accuracy, and maybe most importantly, with the polynomial fit used in extrapolating the data to the thermodynamic limit, it appears that the single-particle gap opens right when magnetic ordering sets in. The only mismatch is at $U / t=3.7$, where we do not detect magnetic ordering but we do detect a small single-particle gap.

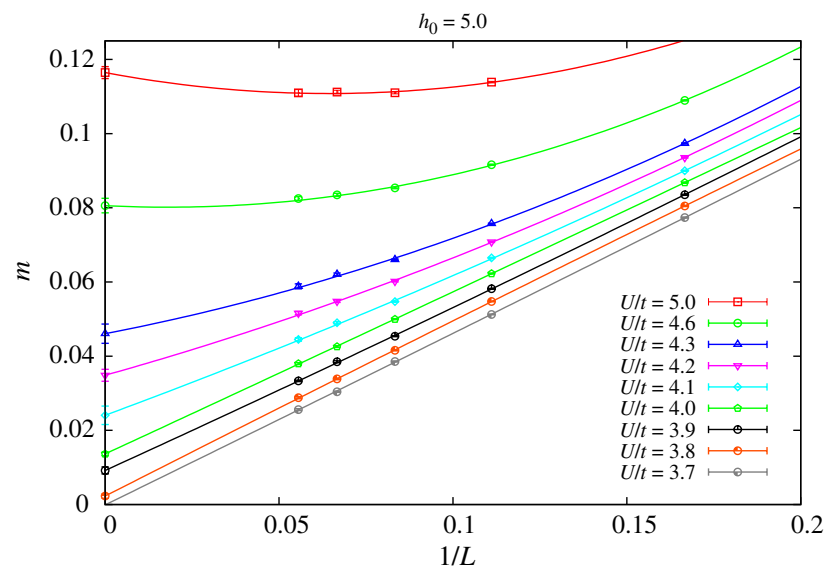

FIG. 3. Magnetic moment at pinning field $h_{0}=5 t$ as a function of $U / t$. Here, we have used $\theta t=320$ and $\Delta \tau t=0.1$. The solid lines are least-squares fits to the form $a+b / L+c / L^{2}$. 


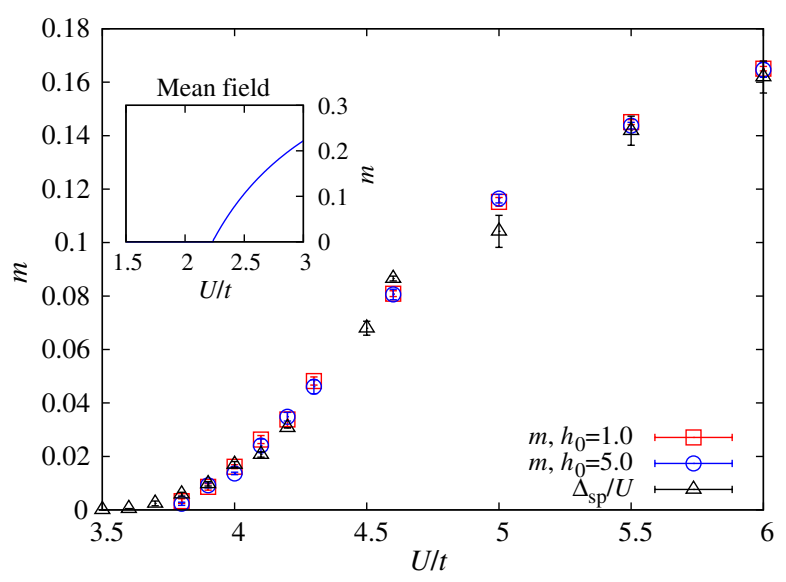

FIG. 4. Staggered moment extrapolated to the thermodynamic limit (see Fig. 3) for two values of the pinning field. We have equally plotted the single-particle gap in units of $U$. The inset plots the staggered magnetization as obtained from a mean-field spin-density-wave ansatz.

(ii) The QMC data in Fig. 4 show that over a wide parameter range, the single-particle gap, measured in units of the Hubbard $U$, tracks the staggered magnetization. We take this fact as a strong indication that the magnetization provides the only relevant scale in the problem, determining directly the single-particle gap. We will see below that this conclusion, based here on a simple, polynomial extrapolation of the finite-size data, is also obtained, if a more refined data analysis is performed.

(iii) The data in Fig. 4 exhibit an unusual inflection point at approximately $U / t=4$.1. Such an inflection point is clearly absent at the mean-field level (see the inset of Fig. 4). We will discuss the implications of this inflection point in the next section. Let us finally note that in previous calculations [1], we were unable to resolve staggered moments lesser than $m \simeq 0.03$. We thereby missed this inflection point in the polynomially extrapolated magnetization curve and concluded the presence of an intermediate phase [21].

\section{Finite-size scaling}

As mentioned above, one of the particularities of the data presented in Fig. 4 is the occurrence of an inflection point at $U / t=4$.1. It is a natural question to ask if this rather peculiar feature may be an artifact of using a simple polynomial fitting procedure that one would indeed expect to fail close to criticality. The polynomial procedure could result in an overestimation of the magnetization in the vicinity of the critical point between the semimetallic and the insulating phases of the Hubbard model. As we explain next, arguments in favor of this conjecture are provided by the large- $N$ treatment of the Gross-Neveu model [5] and the $\epsilon$ expansion around three spatial dimen- sions in the equivalent Gross-Neveu-Yukawa field theory, formulated in Ref. [6]. Given the order parameter exponent $\beta$, as well as the correlation length exponent $\nu$, the staggered magnetization scales as

$$
m \simeq\left|U-U_{c}\right|^{\beta} \simeq \xi^{-\beta / \nu} .
$$

Using the standard scaling laws [22], the exponent $\beta / \nu$ may conveniently be expressed in terms of the anomalous dimension for the order parameter $\eta$ as

$$
\frac{\beta}{\nu} \equiv \frac{1}{2}([d+z]-2+\eta)
$$

where $d+z$ is the effective dimensionality of the system. If we assume that the Lorentz invariance is emergent at the critical point, as it indeed is close to the upper critical dimension $d_{\mathrm{up}}=3$ of the Gross-Neveu-Yukawa theory [6], and maybe even more generally [23], the dynamical critical exponent is $z=1$. If, then, the anomalous dimension of the order parameter is such that $\eta<3-d$, we find that the combination of the exponents $\beta / \nu<1$ and our polynomial fitting procedure in the previous section could very well overestimate the value of the staggered magnetization. In fact, both the large- $N$ approach [5] and the expansion around the upper critical dimension [6] suggest such an overestimation. Within the first order of the expansion in the parameter $\epsilon=3-d$, for example, $\eta=$ $4 \epsilon / 5$, so that

$$
\frac{\beta}{\nu}=1-\frac{\epsilon}{10}+\mathcal{O}\left(\epsilon^{2}\right) .
$$

In two dimensions, then, $\beta / \nu \simeq 0.9$.

To look for the signs of the Gross-Neveu criticality in the Hubbard model, we carry out a finite-size scaling analysis based on the usual scaling form

$$
m=L^{-\beta / \nu} F\left[L^{1 / \nu}\left(U-U_{c}\right)\right] .
$$

Figure 5(a) plots $m L^{\beta / \nu}$ versus $U$ for the magnetization data at the fixed field $h_{0}=5$. [We will omit at this point the second scaling variable $h_{0} L^{y-d}$, since the scaling dimension $y-d=(\epsilon-\eta) / 2 \ll 1$. This second argument of the scaling function, present in principle, is therefore effectively constant at a fixed $h_{0}$, and its inclusion does not visibly affect the quality of scaling. For further discussion of this point, see the Appendix.] As a guide, we have used the first-order $\epsilon$-expansion value of $\beta / \nu=0.9$. Five curves then all cross at a single point $U_{c} / t \simeq 3.8$, thereby providing a first nontrivial indication of the critical point. This value of $U_{c}$ is slightly larger than that obtained with the polynomial fit. The $\epsilon$-expansion value of the correlation length exponent reads [6]

$$
\nu=\frac{1}{2}+\frac{21}{55} \epsilon+\mathcal{O}\left(\epsilon^{2}\right) .
$$



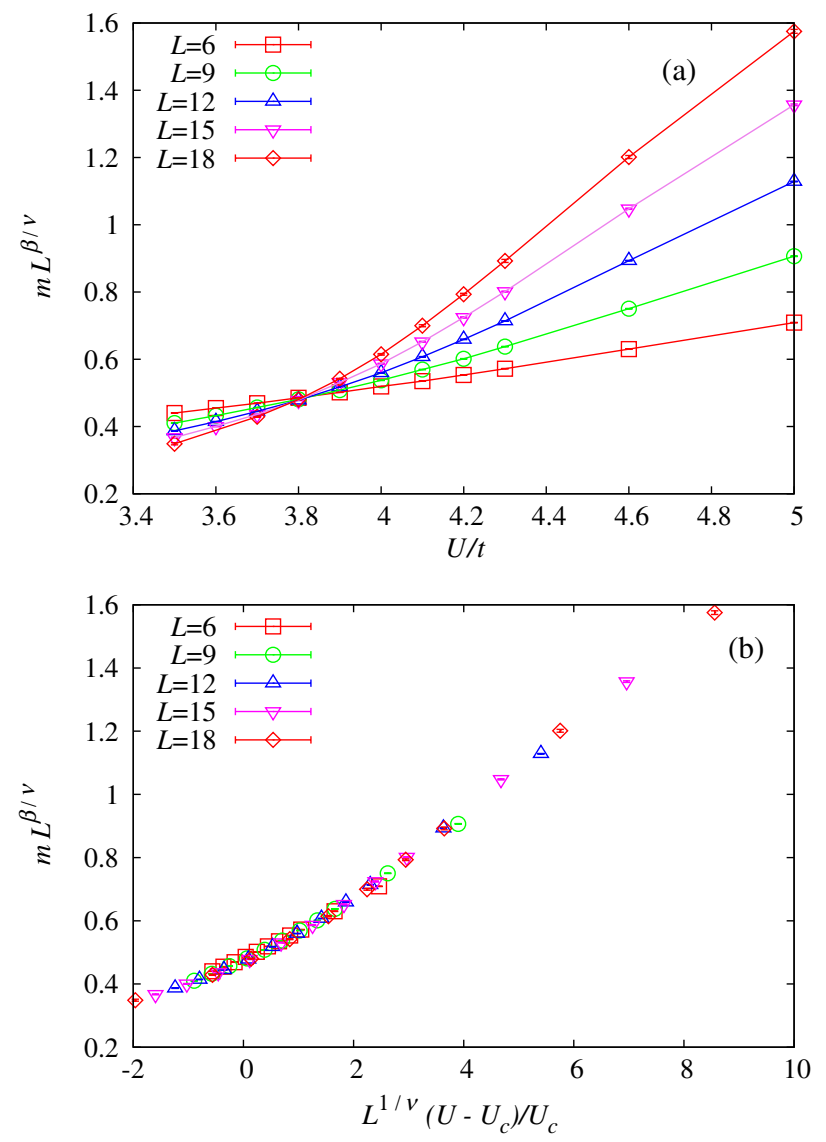

FIG. 5. Data collapse for the magnetization presented in Fig. 3. The exponents are taken for the $\epsilon$ expansion of Ref. [6]. (a) The crossing point pins down the value of $U_{c}$. (b) The data collapse, using $U_{c} / t=3.78$.

With this value of $\nu$, again at $\epsilon=1$, we obtain an excellent data collapse, as is shown in Fig. 5(b).

In accord with the Gross-Neveu-Yukawa theory, the numerical data of Fig. 4 support the interpretation that the magnetization is the only scale in the problem. To further check this interpretation, we have scaled the single-particle gap to the form

$$
\frac{\Delta_{\mathrm{sp}}}{U}=L^{-\beta / \nu} \tilde{F}\left[L^{1 / \nu}\left(U-U_{c}\right)\right]
$$

again using the $\epsilon$-expansion exponents in two dimensions. Figure 6(a) shows that the crossing point of the $\frac{\Delta_{\mathrm{sp}}}{U} L^{\beta / \nu}$ curves again occurs at $U_{c} / t \simeq 3.8$, and Fig. 6(b) shows the collapse. It is quite remarkable that within our precision, the two scaling functions are equal: $\tilde{F}=F$.

Hence, the scaling analysis of our QMC within the Gross-Neveu scenario is consistent with a single continuous quantum phase transition between the semimetal and the antiferromagnetic insulators, and suggests that the firstorder expansion around the upper critical dimension in the
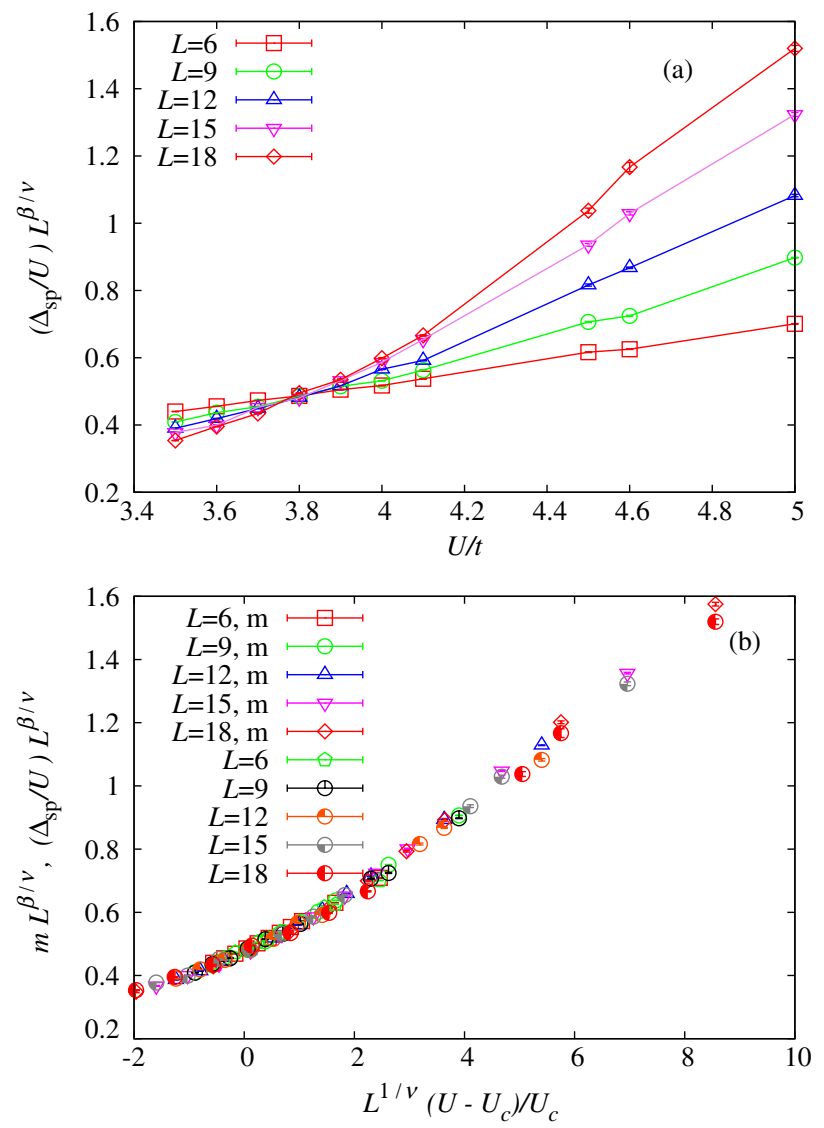

FIG. 6. Data collapse for the single-particle gap. The exponents are taken for the $\epsilon$ expansion of Ref. [6]. (a) The crossing point pins down the value of $U_{c}$. (b) The data collapse again using $U_{c} / t=3.78$. For comparison, we have included the data for the magnetization.

Gross-Neveu-Yukawa theory may already yield rather accurate values of the critical exponents.

\section{DISCUSSION AND CONCLUSIONS}

We have introduced an alternative method to compute the staggered magnetization. By introducing a local magnetic field, we pin the quantization axis of the ordered state. The staggered magnetic moment then corresponds to the local magnetization infinitely far away from the pinning center. The approach has the major advantage that we compute directly the magnetization as opposed to its square when measuring correlation functions. We can therefore expect improved resolution when the local moment is small. One advantage of the approach is an internal cross-check that requires the staggered moment to be independent on the numerical value of the pinning field. We have been able to reach this internal cross-check only in the case of large pinning fields, which is consistent with the approach proposed by Ref. [10], where the pinning field is set to infinity on the boundary of the lattice. If the pinning field is too small, the approach suffers from large 
and nonmonotonic size effects, since the energy scale set by the local magnetic field is unable to overcome the finitesize spin gap. Under these circumstances, the extrapolated value of the magnetization has the tendency of underestimating the order. In this article, we have only considered a very specific form of the pinning field. The number of different choices of pinning fields provides a playground for optimization of the approach and for minimization of the size effects.

The application to the Hubbard model on the honeycomb lattice sheds new light on the phase diagram of this well-known problem. The enhanced precision in comparison to Refs. [1,2] reveals that the staggered moment has the same functional form as the single-particle gap (as measured in units of $U$ ). Remarkably, an excellent data collapse onto a single universal curve is found in the finitesize scaling of both quantities, with the values of the critical exponents characteristic of the Gross-Neveu criticality between the semimetallic and the magnetic insulating phases.

\section{ACKNOWLEDGMENTS}

F.F.A. is very indebted to T. Lang, Z. Y. Meng, A. Muramatsu, and S. Wessel for many valuable comments. We equally acknowledge discussions with A. Chernyshev, L. Fritz, M. Hohenadler, and S. Sorella. This work was initiated at the KITP during the workshop on "Frustrated Magnetism and Quantum Spin Liquids From Theory and Models to Experiments" coordinated by K. Kanoda, P. Lee, A. Vishwanath, and S. White and finalized at the MPI-PKS (Dresden). Funding from the DFG under Grants No. AS120/9-1 and No. AS120/10-1 (Forschergruppe FOR 1807) is acknowledged. I.F.H. has been supported by the NSERC of Canada. We thank the Jülich Supercomputing Centre and the Leibniz-Rechenzentrum in Münich for generous allocation of CPU time.

\section{APPENDIX}

Strictly speaking, the finite-size scaling form for the magnetization in the presence of the external field is

$$
m=L^{-\beta / \nu} G\left(\frac{L}{\xi}, h \xi^{y}\right)=L^{-\beta / \nu} G\left(\frac{L}{\xi}, \frac{h_{0}}{L^{d}} \xi^{y}\right),
$$

where $G(X, Y)$ is the scaling function of two variables, and $\xi$ is the (diverging) correlation length. Here, we have used the fact that the relevant Fourier component of the local pinning field scales as $\frac{h_{0}}{L^{d}}$. The dimension $y$ of the uniform external field is [22]

$$
y=\frac{d+z+2-\eta}{2} .
$$

Away from criticality, $\xi$ is bounded, and in the thermodynamic limit, the magnetization scales to its fieldindependent value. The data in Fig. 1 confirm this feature and show that the extrapolated value of the magnetization is $h_{0}$ independent, provided that for the considered lattice sizes, $h_{0}$ is chosen to be large enough. It is also interesting to point out that for values of $h_{0}$ in the range $1 t<h_{0}<5 t$, the finite-size value of the magnetization is next to independent on the value of the pinning field.

At criticality, we can replace $\xi$ by $L$ to obtain

$$
m=L^{-\beta / \nu} G\left(1, h_{0} L^{y-d}\right) .
$$

With the Lorentz symmetry at the critical point, the value of the dynamical critical exponent is pinned to $z=1$, and the scaling dimension of the local field $h_{0}$ is

$$
y-d=\frac{3-d-\eta}{2} .
$$

In the $\epsilon$ expansion, then,

$$
y-d=\frac{\epsilon}{10}+O\left(\epsilon^{2}\right),
$$

and rather small, presumably even for $\epsilon=1(d=2)$. For a fixed value of $h_{0}$, therefore, the second argument of the scaling function $G$ is almost constant. To be more precise, we can use the asymptotic form $G(1, Y) \propto Y^{1 / \delta}$ with $\frac{1}{\delta}=\frac{\beta}{\nu y}$ [22], such that in the large- $L$

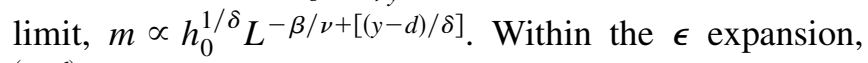
$\frac{(y-d)}{\delta} \simeq 0.05$, which results in a very small correction for considered lattice sizes.

Hence, on the whole, the scaling function $G$ depends rather weakly on the second argument, and for practical purposes, it suffices to neglect it.

[1] Z. Y. Meng, T. C. Lang, S. Wessel, F. F. Assaad, and A. Muramatsu, Quantum Spin Liquid Emerging in TwoDimensional Correlated Dirac Fermions, Nature (London) 464, 847 (2010).

[2] S. Sorella, Y. Otsuka, and S. Yunoki, Absence of a Spin Liquid Phase in the Hubbard Model on the Honeycomb Lattice, Sci. Rep. 2, 992 (2012).

[3] S. Sorella and E. Tosatti, Semi-Metal-Insulator Transition of the Hubbard Model in the Honeycomb Lattice, Europhys. Lett. 19, 699 (1992).

[4] T. Paiva, R. T. Scalettar, W. Zheng, R. R. P. Singh, and J. Oitmaa, Ground-State and Finite-Temperature Signatures of Quantum Phase Transitions in the Half-Filled Hubbard Model on a Honeycomb Lattice, Phys. Rev. B 72, 085123 (2005).

[5] I.F. Herbut, Interactions and Phase Transitions on Graphene's Honeycomb Lattice, Phys. Rev. Lett. 97, 146401 (2006).

[6] I. F. Herbut, V. Juričić, and O. Vafek, Relativistic Mott Criticality in Graphene, Phys. Rev. B 80, 075432 (2009).

[7] H.-Y. Yang, A. F. Albuquerque, S. Capponi, A. M. Läuchli, and K.P. Schmidt, Effective Spin Couplings in the Mott Insulator of the Honeycomb Lattice Hubbard Model, New J. Phys. 14, 115027 (2012). 
[8] C.-C. Chang and R. T. Scalettar, Quantum Disordered Phase near the Mott Transition in the Staggered-Flux Hubbard Model on a Square Lattice, Phys. Rev. Lett. 109, 026404 (2012).

[9] B. K. Clark, Searching for Topological Degeneracy in the Hubbard Model with Quantum Monte Carlo, arXiv:1305.0278.

[10] S. R. White and A. L. Chernyshev, Néel Order in Square and Triangular Lattice Heisenberg Models, Phys. Rev. Lett. 99, 127004 (2007).

[11] M. Hohenadler, T. C. Lang, and F. F. Assaad, Correlation Effects in Quantum Spin-Hall Insulators: A Quantum Monte Carlo Study, Phys. Rev. Lett. 106, 100403 (2011).

[12] M. Hohenadler, Z. Y. Meng, T. C. Lang, S. Wessel, A. Muramatsu, and F. F. Assaad, Quantum Phase Transitions in the Kane-Mele-Hubbard Model, Phys. Rev. B 85, 115132 (2012).

[13] S. Rachel and K. Le Hur, Topological Insulators and Mott Physics from the Hubbard Interaction, Phys. Rev. B 82, 075106 (2010).

[14] F. F. Assaad, Phase Diagram of the Half-Filled TwoDimensional $S U(N)$ Hubbard-Heisenberg Model: A Quantum Monte Carlo Study, Phys. Rev. B 71, 075103 (2005).

[15] M. V. Ulybyshev, P. V. Buividovich, M. I. Katsnelson, and M. I. Polikarpov, Monte Carlo Study of the SemimetalInsulator Phase Transition in Monolayer Graphene with Realistic Interelectron Interaction Potential, Phys. Rev. Lett. 111, 056801 (2013).

[16] Clearly, one cannot exchange the limits in Eq. (6). However, one can measure the magnetization at the largest distance available on the lattice, thereby tying together the thermodynamic and infinite distances from the pinning center limits. This procedure can lead to spurious results.

[17] F. F. Assaad and H. G. Evertz, in Computational ManyParticle Physics, Lecture Notes in Physics Vol. 739, edited by H. Fehske, R. Schneider, and A. Weiße (SpringerVerlag, Berlin, 2008), p. 277-356.

[18] S. Capponi and F. F. Assaad, Spin and Charge Dynamics of the Ferromagnetic and Antiferromagnetic TwoDimensional Half-Filled Kondo Lattice Model, Phys. Rev. B 63, 155114 (2001).

[19] Here, large means comparable to the bandwidth. If the pinning field is much larger than the bandwidth, charge fluctuations on the pinning site will be blocked by an energy scale set by $h_{0}$. Thereby, in the limit $h_{0} \rightarrow \infty$, the pinning site effectively drops out of the Hamiltonian and no symmetry breaking occurs. The very slight drop in the magnetization at $h_{0}=5$ and on small lattices in Fig. 1(a) could be a precursor of this effect.

[20] S. Wessel (private communication).

[21] In Ref. [1], a small but finite spin gap with maximal value at $U / t=4$ was reported. The spin gap is determined with the same method as the single-particle gap, by fitting the tail of the imaginary time-displaced spin-spin correlation functions to a single exponential. Enhancing the imaginary time range for the fit produces a slight decrease of the spin gap for the larger lattices sizes. This slight decrease renders the extrapolation to the thermodynamic limit inconclusive.

[22] I. Herbut, A Modern Approach to Critical Phenomena (Cambridge University Press, Cambridge, England, 2007).

[23] I. F. Herbut, V. Juričić, and B. Roy, Theory of Interacting Electrons on the Honeycomb Lattice, Phys. Rev. B 79, 085116 (2009). 